\title{
On the solvability of the Cauchy problem for linear integral-differential equations
}

\author{
Nataliya Dilna
}




\title{
ON THE SOLVABILITY OF THE CAUCHY PROBLEM FOR LINEAR INTEGRAL-DIFFERENTIAL EQUATIONS
}

\author{
NATALIYA DILNA
}

[Received: February 14, 2004]

\begin{abstract}
New general unique solvability conditions of the Cauchy problem for many-dimensional systems of linear integral differential equations are established. The conditions obtained are optimal in some sense.

Mathematics Subject Classification: 34K06, 34K10, 45J05

Keywords: Integral-differential equation, differential inequality, Cauchy problem
\end{abstract}

\section{INTRODUCTION}

The purpose of this paper is to establish new efficient conditions sufficient for the unique solvability of the Cauchy problem for some classes of many-dimensional systems of linear integral-differential equations.

The proof of the main Theorem 3.1 of Section 3.1 is based on the application of Theorem 2 from [4] (see Theorem 4.2 in this paper) established by using a result of $[\mathbf{5 , 7 ]}$. Note that similar statements were obtained in $[\mathbf{1 , 6}]$ by different methods.

\section{Notation}

The following notation is used.

(i) $\mathbb{R}=(-\infty, \infty), \mathbb{R}_{+}=[0, \infty), \mathbb{N}=\{1,2,3, \ldots\}$.

(ii) $\|x\|:=\max _{1 \leq k \leq n}\left|x_{k}\right|$ for $x=\left(x_{k}\right)_{k=1}^{n} \in \mathbb{R}^{n}$.

(iii) $C\left([a, b], \mathbb{R}^{n}\right)$ is the Banach space of the continuous functions $[a, b] \rightarrow \mathbb{R}^{n}$ equipped with the standard norm

$$
C\left([a, b], \mathbb{R}^{n}\right) \ni u \longmapsto \max _{s \in[a, b]}\|u(s)\| .
$$

(iv) $L\left([a, b], \mathbb{R}^{n}\right)$ is the Banach space of Lebesgue integrable functions $u:[a, b] \rightarrow$ $\mathbb{R}^{n}$ with the norm

$$
L\left([a, b], \mathbb{R}^{n}\right) \ni u \longmapsto \int_{a}^{b}\|u(s)\| d s .
$$

(v) $\mathscr{L}\left(\mathbb{R}^{n}\right)$ is the algebra of square real matrices of dimension $n$. 
(vi) The relation $x \leq_{\vec{\sigma}} y$ for

$$
\vec{\sigma}:=\left(\begin{array}{c}
\sigma_{1} \\
\sigma_{2} \\
\vdots \\
\sigma_{n}
\end{array}\right)
$$

and $\{x, y\} \subset \mathbb{R}^{n}$ means that $\sigma_{k}\left(y_{k}-x_{k}\right) \geq 0$ for all $k=1,2, \ldots, n$. Similarly, the relation $x<_{\vec{\sigma}} y$ means that $\sigma_{k}\left(y_{k}-x_{k}\right)>0$ for every $k=1,2, \ldots, n$. The symbols " $\geq_{\vec{\sigma}}$ " and " $>\vec{\sigma}$ " are defined by analogy.

\section{INITIAL VALUE PROBLEM FOR THE INTEGRAL-DIFFERENTIAL EQUATION}

We consider the system of $n$ linear inhomogeneous integral-differential equations of the form

$$
u^{\prime}(t)=\sum_{j=1}^{N} \int_{a}^{b} H_{j}(t, s) u\left(\omega_{j}(t, s)\right) d s+f(t), \quad t \in[a, b],
$$

with the initial condition

$$
u(\tau)=c,
$$

and the corresponding homogeneous Cauchy problem

$$
\begin{gathered}
u^{\prime}(t)=\sum_{j=1}^{N} \int_{a}^{b} H_{j}(t, s) u\left(\omega_{j}(t, s)\right) d s, \quad t \in[a, b], \\
u(\tau)=0,
\end{gathered}
$$

where $H_{j}:[a, b] \times[a, b] \rightarrow \mathscr{L}\left(\mathbb{R}^{n}\right)$ and $f:[a, b] \rightarrow \mathbb{R}^{n}$ are integrable functions, and $\omega_{j}:[a, b] \times[a, b] \rightarrow[a, b]$ are measurable functions, $j=1,2, \ldots, N$. By a solution of problem (3.1), (3.2) (respectively, (3.3), (3.4)), according to the definition adopted in the modern theory of functional-differential equations [2], an absolutely continuous function $u:[a, b] \rightarrow \mathbb{R}^{n}$ is meant which possesses property (3.2) (respectively, (3.4)) at the point $\tau$ and satisfies relation (3.1) (respectively, (3.3)) almost everywhere on $[a, b]$. Various equations, e. g.,

$$
\begin{gathered}
u^{\prime}(t)=\lambda(t) \int_{a}^{t} r(s) u(h(s)) d s+f(t), \quad t \in(-\infty, \infty), \\
u^{\prime}(t)=\int_{\alpha(t)}^{\beta(t)} r(s) u(\phi(|t-s|)) d s+f(t), \quad t \in(-\infty, \infty),
\end{gathered}
$$

can be rewritten in form (3.1). 


\subsection{Main theorem}

Here, we state the main theorem on the unique solvability of the initial value problem (3.1), (3.2), which can be used to obtain efficient conditions for concrete equations (see, e. g., Corollary 3.6).

The conditions of the Theorem 3.1 below are formulated in terms of the functions obtained by the consecutive application of the linear operation defined by the right-hand side of equation (3.3) to a given function with suitable properties. More precisely, let us fix some absolutely continuous function $y_{0}:[a, b] \rightarrow \mathbb{R}^{n}$ which satisfies the conditions

$$
y_{0}(\tau)=0
$$

and

$$
y_{0}(t)>_{\vec{\sigma}} 0, \quad t \in[a, b] \backslash\{\tau\},
$$

and consider the sequence of functions given by the recurrence relation

$$
y_{k}(t)=\sum_{j=0}^{N} \int_{\tau}^{t}\left(\int_{a}^{b} H_{j}(\xi, s) y_{k-1}\left(\omega_{j}(\xi, s)\right) d s\right) d \xi, \quad t \in[a, b], \quad k=1,2, \ldots
$$

The following general theorem is true.

Theorem 3.1. Let the functions $\omega_{j}:[a, b] \times[a, b] \rightarrow[a, b]$ be measurable and $H_{j}:[a, b] \times[a, b] \rightarrow \mathscr{L}\left(\mathbb{R}^{n}\right), j=1,2, \ldots, N$, be integrable and such that

$$
\sum_{j=1}^{N} \int_{a}^{b} H_{j}(t, s) \vec{\sigma} d s \operatorname{sign}(t-\tau) \geq_{\vec{\sigma}} 0 \quad \text { for a. e. } t \in[a, b]
$$

with some constant vector $\vec{\sigma}=\operatorname{col}\left(\sigma_{1}, \sigma_{2}, \ldots, \sigma_{n}\right),\left\{\sigma_{k} \mid k=1,2, \ldots, n\right\} \subset\{-1,1\}$. Assume also that there exist some real constants $\alpha \in(0,1)$ and $\varrho \in(1,+\infty)$, integers $k \geq 0$ and $r \geq 1$, and a certain absolutely continuous function $y_{0}:[a, b] \rightarrow \mathbb{R}^{n}$ satisfying relations (3.5) and (3.6) such that the integral-differential inequality

$$
\begin{aligned}
{\left[y_{0}^{\prime}(t)-\frac{\varrho^{k+1}}{1-\alpha} \sum_{j=1}^{N}\left(\int _ { a } ^ { b } H _ { j } ( t , s ) \left[\varrho^{r} y_{k+r}\left(\omega_{j}(t, s)\right)\right.\right.\right.} & \\
& \left.\left.\left.-\alpha y_{k}\left(\omega_{j}(t, s)\right)\right] d s\right)\right] \operatorname{sign}(t-\tau) \geq_{\vec{\sigma}} 0
\end{aligned}
$$

holds for almost every $t$ from $[a, b]$.

Then the Cauchy problem (3.3), (3.4) has only the trivial solution, the inhomogeneous Cauchy problem (3.1), (3.2), has a unique solution $u(\cdot)$ for arbitrary $c \in \mathbb{R}^{n}$ and $f \in L\left([a, b], \mathbb{R}^{n}\right)$, and this solution is representable as the uniformly convergent functional series

$$
u(t)=\sum_{k=0}^{\infty} f^{[k]}(t), \quad t \in[a, b],
$$


where, by definition,

$$
f^{[k]}(t):=\sum_{j=1}^{N} \int_{\tau}^{t}\left(\int_{a}^{b} H_{j}(\xi, s) f^{[k-1]}\left(\omega_{j}(\xi, s)\right) d s\right) d \xi, \quad t \in[a, b], k=1,2, \ldots,
$$

and

$$
f^{[0]}(t):=c+\int_{\tau}^{t} f(s) d s, \quad t \in[a, b] .
$$

Moreover, if the vector-function $f$ and vector $c$ satisfy the additional condition

$$
\int_{\tau}^{t} f(s) d s \geq_{\vec{\sigma}}-c
$$

for all t from $[a, b]$, then the unique solution $u(\cdot)$ of the inhomogeneous problem (3.1), (3.2) satisfies the relation

$$
u(t) \geq_{\vec{\sigma}} 0, \quad t \in[a, b] .
$$

We recall that, everywhere in this paper, notation (vi) of Section 2 is used. For example, condition (3.11) means that the inequality

$$
\sigma_{k}\left[c_{k}+\int_{\tau}^{t} f_{k}(s) d s\right] \geq 0
$$

is true for all $t$ from $[a, b]$ and $k=1,2, \ldots, n$.

Remark 3.2. Under the condition of Theorem 3.1, the uniform convergence of the sequence of functions (3.7) to the function equal identically to zero, i. e., to the unique solution of the homogeneous Cauchy problem (3.3), (3.4), follows from the proof of [7, Theorem 2], which theorem implies Theorems 3.1 and 4.2 of [4] used in this paper.

Remark 3.3. Condition (3.9) is optimal in the sense that the assertion of Theorem 3.1, generally speaking, is not true if (3.9) is assumed with $\varrho=1$, i. e., has the form

$$
\begin{aligned}
{\left[(1-\alpha) y_{0}^{\prime}(t)-\sum_{j=1}^{N}\left(\int _ { a } ^ { b } H _ { j } ( t , s ) \left[y_{k+r}\left(\omega_{j}(t, s)\right)\right.\right.\right.} & \\
& \left.\left.\left.-\alpha y_{k}\left(\omega_{j}(t, s)\right)\right] d s\right)\right] \operatorname{sign}(t-\tau) \geq_{\vec{\sigma}} 0 .
\end{aligned}
$$

This statement is justified by the example below.

Example 3.4. Consider the homogeneous integral-differential equation

$$
u^{\prime}(t)=\frac{2 \operatorname{sign}(t-\tau)}{(a-\tau)^{2}+(b-\tau)^{2}} \int_{a}^{b} u(s) d s, \quad t \in[a, b],
$$


where $\tau$ is a given point from $(a, b)$. Clearly, equation (3.14) can be rewritten as (3.3) if we put

$$
\begin{gathered}
n=1, \quad N=1, \\
H_{1}(t, s)=\frac{2 \operatorname{sign}(t-\tau)}{(a-\tau)^{2}+(b-\tau)^{2}}, \quad(t, s) \in[a, b] \times[a, b] .
\end{gathered}
$$

Let us set

$$
y_{0}(t)=|t-\tau|, \quad t \in[a, b] .
$$

Obviously, this function is absolutely continuous and satisfies conditions (3.5) and (3.6) with $n=1$ and $\vec{\sigma}=1$. We construct the sequence of functions $y_{1}, y_{2}, \ldots$ defined by the formula (3.7). In this case, it has the form

$$
\begin{aligned}
y_{k}(t) & =\int_{\tau}^{t} \frac{2 \operatorname{sign}(\xi-\tau)}{(a-\tau)^{2}+(b-\tau)^{2}}\left(\int_{a}^{b} y_{k-1}(s) d s\right) d \xi \\
& =\frac{2|t-\tau|}{(a-\tau)^{2}+(b-\tau)^{2}} \int_{a}^{b} y_{k-1}(s) d s, \quad t \in[a, b] .
\end{aligned}
$$

It is clear that, by virtue of (3.16),

$$
y_{1}(t)=\frac{2|t-\tau|}{(a-\tau)^{2}+(b-\tau)^{2}} \int_{a}^{b}|s-\tau| d s=|t-\tau|, \quad t \in[a, b] .
$$

Arguing by induction, we obtain that

$$
y_{k}(t)=|t-\tau|, \quad t \in[a, b],
$$

for every $k \geq 0$ It is obvious that, for $\vec{\sigma}=1$ and $N, n$, and $H_{1}$ given by formulae (3.15), the equality

$$
\sum_{j=1}^{N} \int_{a}^{b} H_{j}(t, s) \vec{\sigma} d s \operatorname{sign}(t-\tau)=\frac{2}{(a-\tau)^{2}+(b-\tau)^{2}}
$$

is true and, hence, condition (3.8) is fulfilled.

Let us show that condition (3.9) with $\varrho=1$ (i. e., inequality (3.13)) holds for $\vec{\sigma}=1$ and $N, n$, and $H_{1}$ given by formulae (3.15). Indeed, in this case, according to (3.18), the expression in the left-hand side of inequality (3.13) takes the form

$$
\begin{gathered}
{\left[(1-\alpha) y_{0}^{\prime}(t)-\sum_{j=1}^{N}\left(\int_{a}^{b} H_{j}(t, s)\left[y_{k+r}\left(\omega_{j}(t, s)\right)-\alpha y_{k}\left(\omega_{j}(t, s)\right)\right] d s\right)\right] \operatorname{sign}(t-\tau)} \\
=(1-\alpha)\left(\operatorname{sign}(t-\tau)-\frac{2 \operatorname{sign}(t-\tau)}{(a-\tau)^{2}+(b-\tau)^{2}} \int_{a}^{b}|s-\tau| d s\right) \operatorname{sign}(t-\tau) \\
=(1-\alpha)(1-1)=0
\end{gathered}
$$

and, hence, (3.9) is satisfied in the form of an equality. However, the function

$$
u(t)=\lambda|t-\tau|, \quad t \in[a, b]
$$


where $\lambda \in \mathbb{R}$ is arbitrary, is a non-trivial solution of the homogeneous Cauchy problem (3.14), (3.4).

Thus, in Theorem 3.1, one cannot replace condition (3.9) with $\varrho>1$ by condition (3.13) because the latter does not guarantee the unique solvability of the Cauchy problem under consideration.

\subsection{Corollaries}

Conditions (3.5) and (3.6) are satisfied, e. g., for the function

$$
y_{0}(t)=|t-\tau|^{q} \vec{\sigma}, \quad t \in[a, b],
$$

where $q$ is some natural number and $\vec{\sigma}=\left(\sigma_{k}\right)_{k=1}^{n}$ is an $n$-dimensional vector such that $\sigma_{k} \in\{-1,1\}$ for all $k=1,2, \ldots, n$. The following results then follow from Theorem 3.1.

Corollary 3.5. Let the functions $\omega_{j}:[a, b] \times[a, b] \rightarrow[a, b]$ be measurable, $H_{j}:$ $[a, b] \times[a, b] \rightarrow \mathscr{L}\left(\mathbb{R}^{n}\right)$ be integrable, and inequality (3.8) be satisfied for some $\vec{\sigma} \in\{-1,1\}^{n}$. Moreover, assume that there exist a real number $\gamma \in(0,1)$ and some $q \in \mathbb{N}$ such that the inequality

$$
\begin{array}{r}
\sum_{j_{1}=1}^{N} \sum_{j_{2}=1}^{N} \int_{\tau}^{t}\left(\int_{a}^{b} H_{j_{1}}(\xi, p)\left(\int_{\tau}^{\omega_{j_{1}}(\xi, p)}\left(\int_{a}^{b} H_{j_{2}}(\eta, s) \vec{\sigma}\left|\omega_{j_{2}}(\eta, s)-\tau\right|^{q} d s\right) d \eta\right) d p\right) d \xi \\
\leq \sum_{\vec{\sigma}} \gamma \sum_{j_{1}=1}^{N} \int_{\tau}^{t}\left(\int_{a}^{b} H_{j_{1}}(\eta, s) \vec{\sigma}\left|\omega_{j_{1}}(\eta, s)-\tau\right|^{q} d s\right) d \eta
\end{array}
$$

is true for almost every $t \in[a, b]$

Then for arbitrary $c \in \mathbb{R}^{n}$ and $f \in L\left([a, b], \mathbb{R}^{n}\right)$, the inhomogeneous Cauchy problem (3.1), (3.2) is uniquely solvable, and homogeneous Cauchy problem (3.3), (3.4) has only trivial solution. Moreover, if the function $f$ and vector $c$ satisfy inequality (3.11) for all $t \in[a, b]$, then the unique solution of problem (3.1), (3.2) satisfies condition (3.12).

Corollary 3.6. Assume that the functions $\omega_{j}:[a, b] \times[a, b] \rightarrow[a, b]$ are measurable and the integrable $H_{j}:[a, b] \times[a, b] \rightarrow \mathscr{L}\left(\mathbb{R}^{n}\right)$ satisfy (3.8) with some vector $\vec{\sigma} \in\{-1,1\}^{n}$. Let, moreover, there exist some constants $\gamma \in(0,1)$ and $q \in \mathbb{N}$ such that the inequality

$$
\sum_{j=1}^{N} \int_{\tau}^{t}\left[\int_{a}^{b}\left|\omega_{j}(\eta, s)-\tau\right|^{q} H_{j}(\eta, s) d s\right] d \eta \vec{\sigma} \leq_{\vec{\sigma}} \gamma|t-\tau|^{q} \vec{\sigma}
$$

is fulfilled for a. e. $t \in[a, b]$.

Then the conclusion of Corollary 3.5 is true for the inhomogeneous problem (3.1), (3.2) and homogeneous problem (3.3), (3.4). 


\subsection{The CASE OF A TWo-dimensional SYSTEM}

Let us obtain conditions sufficient for the unique solvability of the initial value problem

$$
\begin{gathered}
u_{1}^{\prime}(t)=\int_{a}^{b} h_{11}(t, s) u_{1}\left(\omega_{11}(t, s)\right) d s+\int_{a}^{b} h_{12}(t, s) u_{2}\left(\omega_{12}(t, s)\right) d s+f_{1}(t), \\
u_{2}^{\prime}(t)=\int_{a}^{b} h_{21}(t, s) u_{1}\left(\omega_{21}(t, s)\right) d s+\int_{a}^{b} h_{22}(t, s) u_{2}\left(\omega_{22}(t, s)\right) d s+f_{2}(t), \\
u_{1}(\tau)=c_{1}, \\
u_{2}(\tau)=c_{2},
\end{gathered}
$$

where $t \in[a, b]$, the functions $f_{i}$ and $h_{i j}, i, j=1,2$, are integrable, $\omega_{i j}, i, j=1,2$, are measurable, and $c_{1}$ and $c_{2}$ are arbitrary real constants.

The following assertion is true.

Corollary 3.7. Assume that the functions $\omega_{i j}:[a, b] \times[a, b] \rightarrow[a, b]$ are measurable, $h_{i j}:[a, b] \times[a, b] \rightarrow \mathbb{R}$ are integrable for all $i, j=1,2$, and, moreover, the following conditions are satisfied with certain $\left\{\sigma_{1}, \sigma_{2}\right\} \subset\{-1,1\}$ :

$$
\left(\int_{a}^{b} h_{11}(t, s) d s+\sigma_{1} \sigma_{2} \int_{a}^{b} h_{12}(t, s) d s\right) \operatorname{sign}(t-\tau) \geq 0 \quad \text { for } a . \text { e. } t \in[a, b]
$$

and

$$
\left(\sigma_{1} \sigma_{2} \int_{a}^{b} h_{21}(t, s) d s+\int_{a}^{b} h_{22}(t, s) d s\right) \operatorname{sign}(t-\tau) \geq 0 \quad \text { for a. e. } t \in[a, b] .
$$

Let there exist some constants $\gamma \in(0,1)$ and $q \in \mathbb{N}$ such that the inequalities

$$
\begin{aligned}
\int_{\tau}^{t}\left[\int_{a}^{b} \mid \omega_{1}(\eta, s)\right. & \left.-\left.\tau\right|^{q} h_{11}(\eta, s) d s\right] d \eta \\
& +\sigma_{1} \sigma_{2} \int_{\tau}^{t}\left[\int_{a}^{b}\left|\omega_{2}(\eta, s)-\tau\right|^{q} h_{12}(\eta, s) d s\right] d \eta \leq \gamma|t-\tau|^{q}
\end{aligned}
$$

and

$$
\begin{aligned}
\sigma_{1} \sigma_{2} \int_{\tau}^{t}\left[\int_{a}^{b} \mid \omega_{1}(\eta, s)\right. & \left.-\left.\tau\right|^{q} h_{21}(\eta, s) d s\right] d \eta \\
& +\int_{\tau}^{t}\left[\int_{a}^{b}\left|\omega_{2}(\eta, s)-\tau\right|^{q} h_{22}(\eta, s) d s\right] d \eta \leq \gamma|t-\tau|^{q}
\end{aligned}
$$

hold for almost every $t \in[a, b]$. 
Then the Cauchy problem (3.1), (3.2), (3.3), (3.4) has a unique solution for arbitrary $\left\{f_{1}, f_{2}\right\} \subset L([a, b], \mathbb{R})$ and real $c_{1}, c_{2}$. Furthermore, if the inequalities

$$
\begin{aligned}
& \sigma_{1}\left(\int_{\tau}^{t} f_{1}(s) d s+c_{1}\right) \geq 0, \\
& \sigma_{2}\left(\int_{\tau}^{t} f_{2}(s) d s+c_{2}\right) \geq 0
\end{aligned}
$$

are true for all $t \in[a, b]$, then the unique solution $\left(u_{1}, u_{2}\right)$ of problem (3.1), (3.2), (3.3), (3.4) satisfies the inequalities

$$
\begin{aligned}
& \sigma_{1} u_{1}(t) \geq 0, \\
& \sigma_{2} u_{2}(t) \geq 0
\end{aligned}
$$

for all $t \in[a, b]$.

\section{Proofs of the statements of Sections 3.1 and 3.2}

We recall the following definition [7].

Definition 4.1. An operator $l: C\left([a, b], \mathbb{R}^{n}\right) \rightarrow L\left([a, b], \mathbb{R}^{n}\right)$ is said to be $(\vec{\sigma}, \tau)$ positive with some $\vec{\sigma} \in\{-1,1\}^{n}$ and $\tau \in[a, b]$ if relation (3.12) implies that

$$
(l u)(t) \operatorname{sign}(t-\tau) \geq_{\vec{\sigma}} 0 \quad \text { for a. e. } \quad t \in[a, b] .
$$

In other words, this means that the inequalities

$$
\left(l_{k} u\right)(t) \operatorname{sign}(t-\tau) \geq 0, \quad k=1,2, \ldots, n,
$$

are true for a. e. $t \in[a, b]$ if

$$
\sigma_{k} u_{k}(t) \geq 0, \quad k=1,2, \ldots, n
$$

for all $t \in[a, b]$. Here, $l_{k}: C\left([a, b], \mathbb{R}^{n}\right) \rightarrow L([a, b], \mathbb{R}), k=1,2, \ldots, n$, are the components of the operator $l: C\left([a, b], \mathbb{R}^{n}\right) \rightarrow L\left([a, b], \mathbb{R}^{n}\right)$.

We need the following theorem on the unique solvability of the Cauchy problem established in $[\mathbf{3 , 4}]$.

Theorem 4.2. Let us suppose that the linear operator $l$ in the equation

$$
u^{\prime}(t)=(l u)(t)+f(t), \quad t \in[a, b],
$$

is $(\vec{\sigma}, \tau)$-positive for some $\vec{\sigma} \in\{-1,1\}^{n}$. Assume also that there exist real constants $\alpha \in(0,1)$ and $\varrho \in(1,+\infty)$, integers $k \geq 0$ and $r \geq 1$, and an absolutely continuous function $y_{0}:[a, b] \rightarrow \mathbb{R}^{n}$ with properties (3.5) and (3.6) such that the integraldifferential inequality

$$
\left[y_{0}^{\prime}(t)-\frac{\varrho^{k+1}}{1-\alpha} l\left(\varrho^{r} y_{k+r}-\alpha y_{k}\right)(t)\right] \operatorname{sign}(t-\tau) \geq_{\vec{\sigma}} 0
$$

is satisfied for almost every t from $[a, b]$. 
Then the homogeneous Cauchy problem

$$
\begin{gathered}
u^{\prime}(t)=(l u)(t), \quad t \in[a, b], \\
u(\tau)=0
\end{gathered}
$$

has only the trivial solution, and the corresponding inhomogeneous problem (4.1), (3.2) is uniquely solvable for arbitrary $c \in \mathbb{R}^{n}$ and $f \in L\left([a, b], \mathbb{R}^{n}\right)$. The unique solution $u(\cdot)$ of problem (4.1), (3.2) is, moreover, representable as the uniformly convergent functional series (3.10).

If, in addition, $c$ and $f$ satisfy condition (4.1), then the above-mentioned solution $u(\cdot)$ possesses property $(3.12)$.

\subsection{Proof of Theorem 3.1}

The assertion of Theorem 3.1 follows from Theorem 2 of [4], which is formulated here as Theorem 4.2, because assumption (3.8) guarantees that the linear operator

$$
C\left([a, b], \mathbb{R}^{n}\right) \ni u \longmapsto l u:=\sum_{j=1}^{N} \int_{a}^{b} H_{j}(\cdot, s) u\left(\omega_{j}(\cdot, s)\right) d s
$$

is $(\vec{\sigma}, \tau)$-positive in the sense of Definition 4.1. Indeed, the following lemma is true.

Lemma 4.3. For arbitrary measurable functions $\omega_{j}:[a, b] \times[a, b] \rightarrow[a, b]$ and integrable functions $H_{j}:[a, b] \times[a, b] \rightarrow \mathscr{L}\left(\mathbb{R}^{n}\right), j=1,2, \ldots, N$, satisfying condition (3.8) with some column $\vec{\sigma} \in\{-1,1\}^{n}$, the linear operator (4.4) is $(\vec{\sigma}, \tau)$ positive.

Proof. Let us assume that a function $u \in C\left([a, b], \mathbb{R}^{n}\right)$ satisfies condition (3.12). Consider the expression

$$
\begin{aligned}
\sigma_{k} \sum_{j=1}^{N} \int_{a}^{b} H_{j}(t, s) u\left(\omega_{j}(t, s)\right) d s \operatorname{sign}(t-\tau) \\
\quad=\sigma_{k} \sum_{j=1}^{N} \sum_{v=1}^{n} \int_{a}^{b} \sigma_{v} h_{k v}^{j}(t, s) \operatorname{sign}(t-\tau) \sigma_{v} u_{v}\left(\omega_{j}(t, s)\right) d s, \quad t \in[a, b],
\end{aligned}
$$

where $h_{k v}^{j}, k, v=1,2, \ldots, n$, are the corresponding coordinates of the matrix $H_{j}$, $j=1,2, \ldots, N$, i. e.,

$$
H_{j}(t, s)=\left(\begin{array}{ccc}
h_{11}^{j}(t, s) & \ldots & h_{1 n}^{j}(t, s) \\
\ldots & \ldots & \ldots \\
h_{n 1}^{j}(t, s) & \ldots & h_{n n}^{j}(t, s)
\end{array}\right), \quad(t, s) \in[a, b]^{2} .
$$

Every $k$ th component of the vector

$$
\sum_{j=1}^{N} \int_{a}^{b} H_{j}(t, s) \vec{\sigma} d s \operatorname{sign}(t-\tau)
$$


has the form

$$
\sum_{j=1}^{N} \sum_{v=1}^{n} \int_{a}^{b} \sigma_{v} h_{k v}^{j}(t, s) d s \operatorname{sign}(t-\tau)
$$

and, therefore, by (3.8),

$$
\sigma_{k} \sum_{j=1}^{N} \sum_{v=1}^{n} \int_{a}^{b} \sigma_{v} h_{k v}^{j}(t, s) d s \operatorname{sign}(t-\tau) \geq 0
$$

for all $k$. Thus, in view of (3.12) and (4.5),

$$
\operatorname{diag}\left(\sigma_{1}, \sigma_{2}, \ldots, \sigma_{n}\right) \sum_{j=1}^{N} \int_{a}^{b} H_{j}(t, s) u\left(\omega_{j}(t, s)\right) d s \operatorname{sign}(t-\tau) \geq 0
$$

componentwise, i. e.,

$$
\sum_{j=1}^{N} \int_{a}^{b} H_{j}(t, s) u\left(\omega_{j}(t, s)\right) d s \operatorname{sign}(t-\tau) \geq_{\vec{\sigma}} 0
$$

for a. e. $t \in[a, b]$.

Since $u$ is an arbitrary function satisfying condition (3.12), we have shown that operator (4.4) is $(\vec{\sigma}, \tau)$-positive in the sense of Definition 4.1.

\subsection{Proof of Corollary 3.5}

To prove Corollary 3.5, it is sufficient to apply Corollary 5 from [4] putting $k=1$ and $r=1$ and defining the function $y_{0}$ by formula (3.21).

\subsection{Proof of Corollary 3.6}

Corollary 3.6 follows from Corollary 6 of [4]. Inequality (3.23) is fulfilled for operator (4.4) and the function $y_{0}$ given by formula (3.21). Indeed, it is easy to verify that the derivative $y_{0}^{\prime}$ of function (3.21) is given by the formula

$$
y_{0}^{\prime}(t)=q|t-\tau|^{q-1} \operatorname{sign}(t-\tau), \quad t \in[a, b] .
$$

Therefore,

$$
y_{0}^{\prime}(t) \operatorname{sign}(t-\tau) \geq_{\vec{\sigma}} 0, \quad t \in[a, b],
$$

because, obviously,

$$
q|t-\tau|^{q-1} \vec{\sigma} \geq_{\vec{\sigma}} 0, \quad t \in[a, b] .
$$




\subsection{Proof of Corollary 3.7}

Let us put $n=2$ and $N=4$ in equation (3.1) and define the matrix-valued functions $H_{j}, 1 \leq j \leq 4$, by the formulae

$$
\begin{array}{ll}
H_{1}(t, s)=\left(\begin{array}{cc}
h_{11}(t, s) & 0 \\
0 & 0
\end{array}\right), & H_{2}(t, s)=\left(\begin{array}{cc}
0 & h_{12}(t, s) \\
0 & 0
\end{array}\right), \\
H_{3}(t, s)=\left(\begin{array}{cc}
0 & 0 \\
h_{21}(t, s) & 0
\end{array}\right), & H_{4}(t, s)=\left(\begin{array}{cc}
0 & 0 \\
0 & h_{22}(t, s)
\end{array}\right)
\end{array}
$$

for a. e. $t$ and $s$ from $[a, b]$. Let $\omega_{1}=\omega_{11}, \omega_{2}=\omega_{12}, \omega_{3}=\omega_{21}$, and $\omega_{4}=\omega_{22}$. Then, as is easy to see, the inhomogeneous Cauchy problem (3.24), (3.25), (3.26), (3.27) takes form (3.1), (3.2). Applying Corollary 3.6, we obtain the required assertion.

\section{REFERENCES}

[1] Agarwal, R. P. and Ronto, A.: Linear functional differential equations possessing solutions with a given growth rate, J. Inequalities Appl., to appear.

[2] Azbelev, N., Maksimov, V., and Rakhmatullina, L.: Introduction to the Theory of Linear Functional Differential Equations, vol. 3 of Advanced Series in Mathematical Science and Engineering, World Federation Publishers Company, Atlanta, GA, 1995.

[3] Dilnaya, N. Z. and Ronto, A. N.: On the solvability of the Cauchy problem for systems of linear functional differential equations with $(\vec{\sigma}, \tau)$-positive right hand sides, Dopov. Nats. Akad. Nauk Ukr. (2004), No. 2, 29-35.

[4] Dilnaya, N. Z. and Ronto, A. N.: Some new solvability conditions of the Cauchy problem for systems of linear functional differential equations, Ukrain. Math. J., 56 (2004), No. 7, 867-884.

[5] Ronto, A.: New differential inequalities properties of whose solutions ensure the solvability of the Cauchy problem for linear functional differential equations, Dopov. Nats. Akad. Nauk Ukr. (2004), No. 1, 26-32.

[6] Rontó, A. And Šremr, J.: Abstract differential inequalities and the Cauchy problem for infinitedimensional linear functional differential equations, Archives Inequalities Appl., to appear.

[7] RonTo, A. N.: Exact solvability conditions of the Cauchy problem for systems of linear firstorder functional differential equations determined by $\left(\sigma_{1}, \sigma_{2}, \ldots, \sigma_{n} ; \tau\right)$-positive operators, Ukrain. Math. J., 55 (2003), No. 11, 1853-1884.

\section{Author's Address}

\section{Nataliya Dilna:}

Institute of Mathematics, National Academy of Sciences of Ukraine, 3 Tereschenkovskaya St., $01601 \mathrm{KIEv}$, UKRAINE

E-mail address: dilna@imath.kiev.ua 